\title{
Towards active electro-optic lithium niobate metasurfaces
}

\author{
Flavia Timpu ${ }^{1}$, Helena Weigand $^{1}$, Fabian Kaufmann ${ }^{1}$, Felix U. Richter ${ }^{1}$, Viola-Valentina \\ Vogler-Neuling ${ }^{1}$, Artemios Karvounis ${ }^{1}$, and Rachel Grange ${ }^{1 *}$ \\ ${ }^{1}$ ETH Zurich, Institute for Quantum Electronics, Department of Physics, Auguste-Piccard-Hof 1, 8093 \\ Zurich, Switzerland
}

\begin{abstract}
We present the design and fabrication advances on active lithium niobate metasurfaces. We determine by numerical calculations a metasurface design with electro-magnetic resonances in the visible and near-infrared, by taking into account the constraints for fabrication on thin films of lithium niobate. We suggest that the optical properties of the metasurface can be switched using the electro-optical properties of lithium niobate.
\end{abstract}

\section{Metasurface design for electro-optic switching}

We design metasurfaces of lithium niobate $\left(\mathrm{LiNbO}_{3}, \mathrm{LNO}\right)$ starting from a monocrystalline $200 \mathrm{~nm}$ layer on $500 \mu \mathrm{m}$ quartz. LNO is a non-centrosymmetric metal-oxides with a high refractive index, second-order susceptibility tensor and electro-optic coefficients [1]. By nanostructuring LNO at the sub-wavelength scale, it is possible to control the optical properties by tuning the confinement of light in the material.
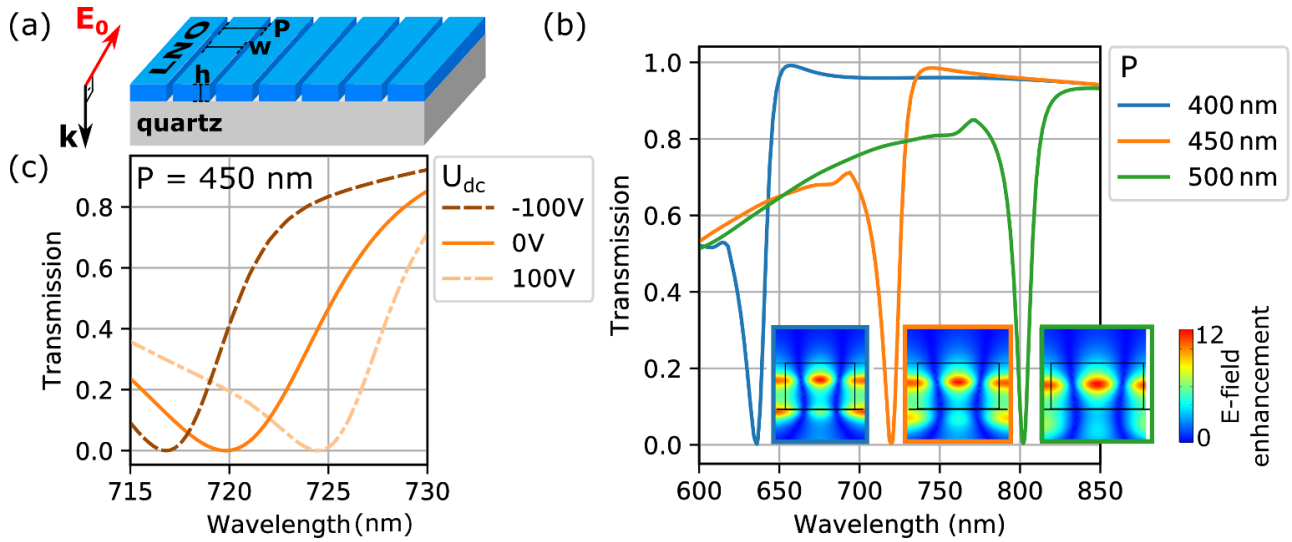

Figure 1. (a) Schematic of LNO gratings on a quartz substrate: period $P$, width $w=P-100 \mathrm{~nm}$, height $h=200 \mathrm{~nm}$. (b) Calculated transmittance of a plane wave polarized along the gratings at normal incidence. The insets show the electric field enhancement at the transmission resonance in a plane perpendicular to the grating. (c) Calculated transmission under actuation with a DC-bias voltage for the LNO grating with a period of $450 \mathrm{~nm}$.

In Figure 1(a), we present a basic design of LNO gratings on a quartz substrate. For a well-defined wavelength that increases with the period, we observe the total suppression of the transmitted light (Fig. 1(b)). At these wavelengths, the electric field is enhanced up to 12 times compared to an unpatterned layer of LNO.

We further show that the application of an electrical voltage $U$ can actively tune the optical transmittance of the LNO gratings. We assume that the extraordinary optical axis of LNO and

\footnotetext{
*e-mail: grange@phys.ethz.ch
} 
the external DC-bias field are oriented along the gratings. Thus, the extraordinary refractive index $n_{e}$ changes due to $U$ and due to the field enhancement factor, defined as the average field enhancement in the volume of LNO [2]. We estimate a $5 \mathrm{~nm}$ shift of the transmission resonance for $\mathrm{U}=100 \mathrm{~V}$ (Fig. 1(c)).

\section{Fabrication approach and preliminary measurements}

We verify our numerical results by fabricating LNO gratings on quartz using focused ion beam (FIB) milling. We measure their optical transmission in an optical setup described elsewhere [3]. For all three LNO gratings with periods from $400 \mathrm{~nm}$ to $500 \mathrm{~nm}$, we observe a transmission resonance at wavelengths that increase with their period. In comparison to the simulation, the wavelength of the transmission resonance is red shifted due to geometry differences between simulated and fabricated gratings. Furthermore, the reduced suppression of the measured transmission is a consequence of the measurement setup, which includes not only the normal-incident wave, but several angles of incidence [3].

In addition to the fabrication by FIB, we are currently developing a top-down fabrication approach using e-beam and inductively coupled plasma (ICP/RIE) etching (Fig. 2 (c)) to improve the fabrication yield. Preliminary fabrication results of a LNO metasurface consisting of holes (Fig. 2 (d)) are promising.

(a)

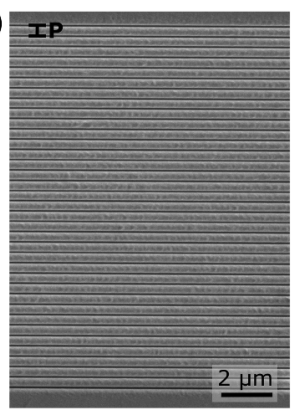

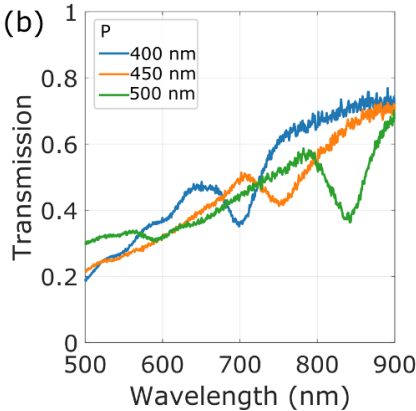

(c)
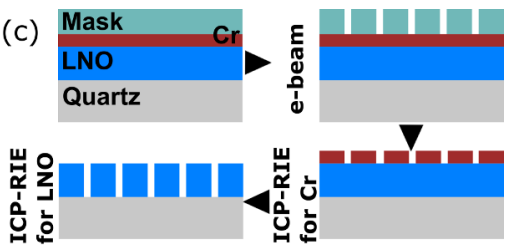

(d)

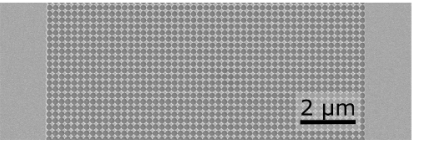

Figure 2. (a) SEM image of LNO gratings fabricated by FIB. (b) Measured transmission of the LNO gratings. (c) Schematic flow of the ICP fabrication protocol of LNO metasurfaces. (d) SEM image of a metasurface after the ICP-RIE for chromium $(\mathrm{Cr})$.

\section{Conclusions}

We show initial designs of LNO periodic arrays with efficient electro-optical switching. We demonstrate the fabrication of such gratings by FIB and we propose more efficient fabrication by ICP dry etching. Preliminary optical measurements show good agreement with the simulation. In a next step, more complex designs will be produced by ICP. Furthermore, the electro-optical switching will be measured.

\section{References}

[1] M.J. Weber, Handbook of optical materials (CRC Press LLC, 2003)

[2] Z. Huang, H. Lu, H. Xiong, Y. Li, H. Chen, W. Qiu, H. Guan, J. Dong, W. Zhu, J. Yu et al., Nanomaterials 9, 69 (2019)

[3] F. Timpu, M. Reig Escalé, M. Timofeeva, N. Strkalj, M. Trassin, M. Fiebig, R. Grange, Advanced Optical Materials 7, 1900936 (2019) 